\title{
Simulations of ultrafast $x$-ray laser experiments
}

C. Fortmann-Grote

A. A. Andreev

K. Appel

J. Branco

R. Briggs

M. Bussmann

A. Buzmakov

M. Garten

A. Grund

A. Huebl

Z. Jurek

N. D. Loh

M. Nakatsutsumi

L. Samoylova

R. Santra

E. A. Schneidmiller

A. Sharma

K. Steiniger 


\title{
Simulations of ultrafast $x-$ ray laser experiments
}

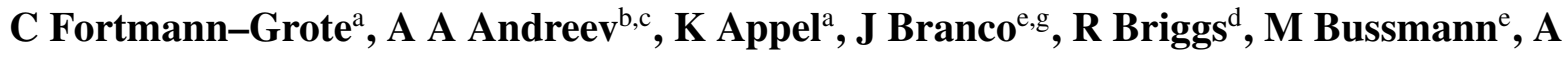

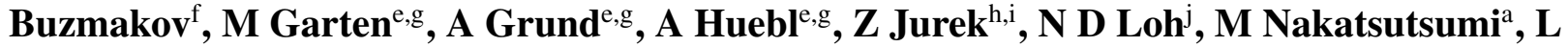 \\ Samoylova $^{\mathrm{a}}$, R Santra ${ }^{\mathrm{h}, \mathrm{i}, \mathrm{k}}$, E A Schneidmiller ${ }^{1}$, A Sharma ${ }^{\mathrm{c}}$, K Steiniger ${ }^{\mathrm{e}, \mathrm{g}}$, S Yakubov ${ }^{\mathrm{l}}$, C H \\ Yoon $^{\mathrm{m}}$, M V Yurkov ${ }^{1}$, U Zastrau $^{\mathrm{a}}$, B Ziaja-Motyka ${ }^{\mathrm{h}, \mathrm{i}, \mathrm{n}}$, A P Mancuso ${ }^{\mathrm{a}}$ \\ ${ }^{a}$ European XFEL GmbH, Holzkoppel 4, 22869 Schenefeld, Germany \\ ${ }^{b}$ Max Born Institute, Berlin, Max-Born-Straße 2 A, 12489 Berlin, Germany \\ ${ }^{c}$ ELI ALPS, Tisza Lajos Krt. 85-87, H-6722 Szeged, Hungary \\ ${ }^{\mathrm{d}}$ European Synchrotron Radiation Facility ESRF, 71, Avenue des Martyrs, CS 40220, 38043 Grenoble, Cedex 9 , \\ France \\ ${ }^{\mathrm{e}}$ Helmholtz-Zentrum Dresden-Rossendorf, P.O. Box 510119, 01314 Dresden, Germany \\ ${ }^{\mathrm{f}}$ FSRC "Crystallography and Photonics" Russian Academy of Sciences, Moscow, Russia \\ ${ }^{\mathrm{g}}$ Technische Universität Dresden, 01062 Dresden, Germany \\ ${ }^{\mathrm{h}}$ Center for Free Electron Laser Science, Deutsches Elektronen Synchrotron, Notkestrasse 85, 22607 Hamburg, \\ Germany \\ ${ }^{\mathrm{i}}$ The Hamburg Center for Ultrafast Imaging, Luruper Chaussee 149, 22761 Hamburg, Germany \\ ${ }^{\mathrm{j}}$ Department of Physics, National University of Singapore, Singapore \\ ${ }^{\mathrm{k}}$ Department of Physics, University of Hamburg, Jungiusstrasse 9, 20355 Hamburg, Germany \\ ${ }^{1}$ Deutsches Elektronen Synchrotron, Notkestrasse 85, 22607 Hamburg, Germany \\ ${ }^{\mathrm{m}}$ Linac Coherent Light Source, SLAC National Accelerator Laboratory, 2575 Sand Hill Road, Menlo Park CA \\ 94025, USA \\ ${ }^{\mathrm{n}}$ Institute of Nuclear Physics, Polish Academy of Sciences, Radzikowskiego 152, 31-342 Krakow, Poland
}

\begin{abstract}
Simulations of experiments at modern light sources, such as optical laser laboratories, synchrotrons, and free electron lasers, become increasingly important for the successful preparation, execution, and analysis of these experiments investigating ever more complex physical systems, e.g. biomolecules, complex materials, and ultra-short lived states of matter at extreme conditions. We have implemented a platform for complete start-to-end simulations of various types of photon science experiments, tracking the radiation from the source through the beam transport optics to the sample or target under investigation, its interaction with and scattering from the sample, and registration in a photon detector. This tool allows researchers and facility operators to simulate their experiments and instruments under real life conditions, identify promising and unattainable regions of the parameter space and ultimately make better use of valuable beamtime. In this paper, we present an overview about status and future development of the simulation platform and discuss three applications: 1.) Single-particle imaging of biomolecules using $x-$ ray free electron lasers and optimization of $\mathrm{x}$-ray pulse properties, 2.) $\mathrm{x}$-ray scattering diagnostics of hot dense plasmas in high power laser-matter interaction and identification of plasma instabilities, and 3.) $x$-ray absorption spectroscopy in warm dense matter created by high energy laser-matter interaction and pulse shape optimization for low-isentrope dynamic compression.
\end{abstract}

Keywords: Start-to-end simulations, single-particle imaging, radiation damage, high power lasers, warm dense matter.

*C. Fortmann-Grote carsten.grote@xfel.eu

\section{Introduction}

The computer program simex_platform ${ }^{1,2}$ is a modular software environment for the simulation of experiments at advanced laser light sources. Users can assemble a virtual experiment

Advances in X-ray Free-Electron Lasers Instrumentation IV, edited by Thomas Tschentscher, Luc Patthey, Proc. of SPIE Vol. 10237, 102370S · (C) 2017 SPIE · CCC code: 0277-786X/17/\$18 · doi: 10.1117/12.2270552 
through the combination of suitable simulation codes for the various instrumental parts of the experiment: creation of the radiation in the light source (e.g. synchrotron radiation (SR) source, free-electron laser (FEL), or optical laser (OL)), propagation of the radiation from the source to the point of interaction with a sample or target, interaction of the light with the sample, propagation of the scattered radiation and other products from the photon-matter interaction (emitted photons, electrons, ions) after the interaction, and detection. We have equipped simex_platform with a number of such codes and corresponding scriptable user interfaces to ease setup and execution of simulation runs. A lightweight abstraction mechanism and interface templates makes the integration of further 3rd party simulation codes straightforward. This allows users and developers of specialized photon science simulation codes to embed their applications into a more realistic simulation environment compared to running the codes isolated with more or less idealized parameters and initial conditions.

\subsection{Simulation Calculators}

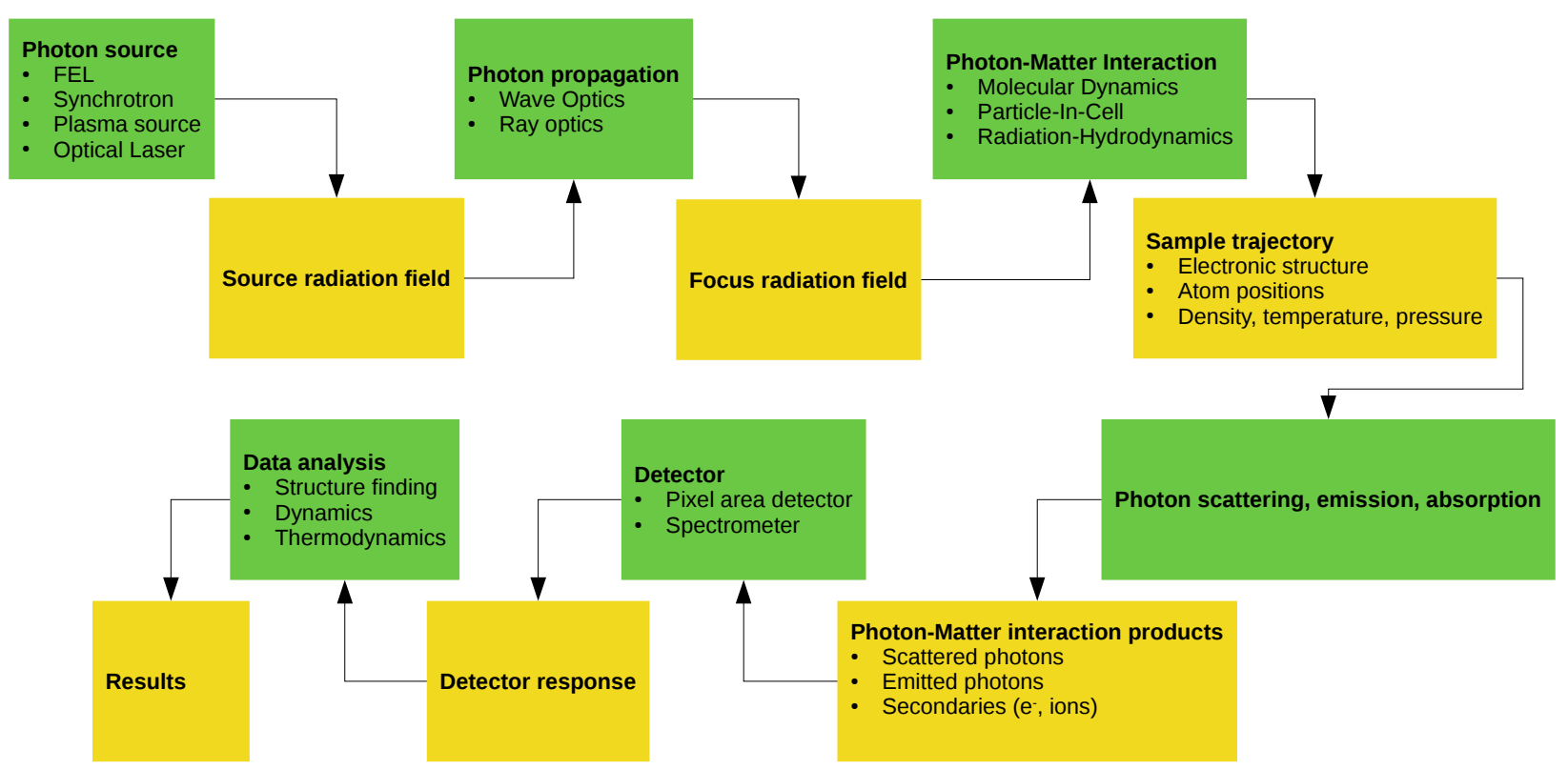

Fig 1 Baseline workflow in simex_plat form. Green boxes represent Calculators, yellow boxes are data interfaces. 
Our software currently supports simulation of photon experiments that follow a sequential base pattern: The radiation is produced in a photon source, propagated through a beamline, it interacts with a sample (target), and photons (or other particles) leaving the sample after scattering or emission are registered in a detector. This linear progression is depicted in Fig. 1. A typical example is an $\mathrm{X}$-ray diffraction experiment: $\mathrm{X}$-ray photons delivered by a SR source or a $\mathrm{X}$-ray free-electron laser (XFEL) and focussed onto a sample by means of focussing x-ray optics, scatter from a sample (e.g. a crystal), and the scattered photons are registered in an area detector behind the sample. Other baseline applications are, e.g., small and wide angle scattering, inelastic $\mathrm{x}$-ray scattering, or x-ray absorption spectroscopy.

Simulation steps are represented by Calculators, depicted as green boxes in Fig. 1. Calculators are classes written in the python programming language ${ }^{3,4}$ and provide scriptable user interfaces to the underlying simulation codes (referred to as Backengines in the following), which perform the actual mathematical and numerical operations on the Calculator's input data.

Each Calculator stores its results in a well defined data format based on the Hierarchical Data Format (hdf5). ${ }^{5}$ Our format is compatible with or convertible into the format used by the framework simS2E, ${ }^{6,7}$ from which simex_platform was derived. In parallel, we also support the “Open Standard for Particle-Mesh data" (openPMD). ${ }^{8}$ These data containers, which may exist as files on disk or as objects in memory are the data interfaces (yellow boxes) in Fig. 1. Output from one Calculator is input the next Calculator.

\section{Single-particle coherent diffractive imaging}

Single-particle imaging (SPI) through coherent diffraction of $\mathrm{x}$-rays from single particles (e.g. biomolecules or clusters) is a $\mathrm{x}$-ray experimental technique to determine the structure of these 
objects without the need for cystallization and thus enables characterization of samples that cannot be crystallized (such as many membrane proteins) and in their natural solvent.

SPI is made possible by XFELs which deliver ultra-short and intense pulses of $\mathrm{x}$-rays onto the sample. While the high photon fluence is needed to compensate for the inherently weak scattering efficiency of these non-crystalline samples, pulse durations of the order of a few femtoseconds allow to probe the sample before it is destroyed in the radiation field..$^{9-13}$

\subsection{Experimental scenario}

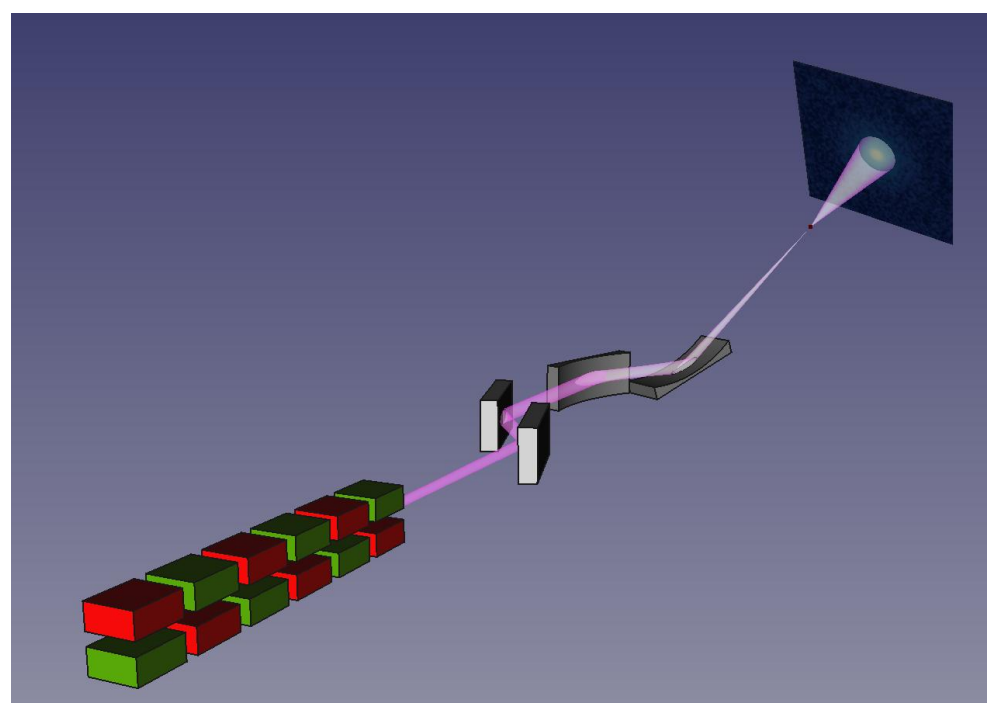

Fig 2 Schematic setup of a single particle imaging experiment

In a SPI experiment, schematically shown in Fig. 2, $\mathrm{x}$-rays are focussed to sub-micrometer spot size onto the sample molecule and scattered photons are recorded in a 2D pixel area detector. After collecting a large number of single-shot diffraction patterns from individual samples and assembly of these patterns into a 3D diffraction volume, ${ }^{14}$ the $3 \mathrm{D}$ electron density distribution can be reconstructed using phasing algorithms, ${ }^{15}$ similar to crystallographic structure determination. 


\subsection{Simulations}

Our simulations track the $\mathrm{x}$-ray field from their production in the undulator section of the freeelectron laser, and propagation through focussing $\mathrm{x}$-ray optics to the sample position. We then simulate the interaction of $\mathrm{x}$-ray photons with the randomly oriented sample and the scattering in a time-resolved manner. The detector signal is calculated from the number of scattered photons at a given pixel position, integrated over the duration of the $\mathrm{x}$-ray pulse. We collect $\approx 10^{5}$ simulated diffraction patterns to sample the source fluctuations, random orientation of the sample molecule and the stochastic nature of photon-matter interaction processes. We feed all simulated patterns into an orientation algorithm, ${ }^{14}$ which assembles a 3D diffraction volume. Finally, an iterative phasing algorithm ${ }^{14,15}$ reconstructs the $3 \mathrm{D}$ electron density, which can be compared to the original sample structure, e.g. to assess the quality of orientation and phasing under given experimental conditions taken into account in the simulations.

\subsection{XFEL source and wavefront propagation}

We simulate the propagation of coherent $\mathrm{x}$-ray photons from the exit of the XFEL's undulator to the sample interaction zone by means of coherent wavefront propagation, ${ }^{16,17}$ assuming that the $\mathrm{x}-$ ray beam is perfectly transverse coherent. The radiation field is represented by two 3 dimensional data arrays for the vertically and horizonally polarized components of the complex electric field at discrete beam-transverse coordinates $x, y$ and at discrete times $t$ at a fixed longitudinal coordinate $z$ along the beamline axis. The simulation starts by reading a file from the XFEL Photon pulses database (XPD) ${ }^{18}$ which contains precomputed fields at the undulator exit $z=0$ for various electron bunch charges, photon energies, and undulator lengths. The database was populated using the FEL simulation code FAST. ${ }^{19}$ Within simex_platform, it is also possible to calculate the FEL 
source with the code Genesis. ${ }^{20}$

The propagation through the beamline is then performed with the software $W P G,{ }^{21}$ a python interface to SRW. ${ }^{22}$ Each element of the beamline, e.g. free space drifts, apertures, lenses, plane and curved mirrors, and gratings, are represented by numerical propagators acting on the radiation field in the frequency domain, either in a multiplicative way (thin optical elements) or by convolution (thick element).

Fig. 3 shows the $\mathrm{x}$-ray intensity distribution, temporal profile, and spectrum before and after propagation of a pulse with $4.96 \mathrm{keV}$ photon energy, $\approx 3 \mathrm{fs}$ pulse duration and $1 \mathrm{~mJ}$ pulse energy $\left(\approx 10^{11}\right.$ photons $)$

\subsection{Photon-Matter Interaction}

Resuls of the photon propagation simulation are picked up by the Photon-matter interaction Calculator to simulate the interaction of $\mathrm{x}$-ray photons with the sample on a microscopic basis.

We use the simulation package XRAYPAC containing the codes XMDYN ${ }^{23,24}$ and XATOM ${ }^{25}$ for classical Molecular Dynamics (MD) propagation of the sample and calculation of ionization, recombination, and scattering cross sections, respectively. At each time step of the propagated x-ray pulse, the code updates the electronic configuration of all atoms by solving a coupled system of rate equations. Transition rates and scattering cross-sections in the presence of the intense $\mathrm{x}$-ray field are calculated from time-dependent perturbation theory with self-consistent Hartree-Fock-Slater atomic orbitals. Atoms and free electrons are propagated according to Newtonian mechanics and classical pairpotentials. The code describes photo-ionization, Auger processes, fluorescence, and three-body recombination, as well as electron-impact ionization and Compton scattering and has

successfully been benchmarked against experimental results. ${ }^{23,26-28}$ The resulting data contains 

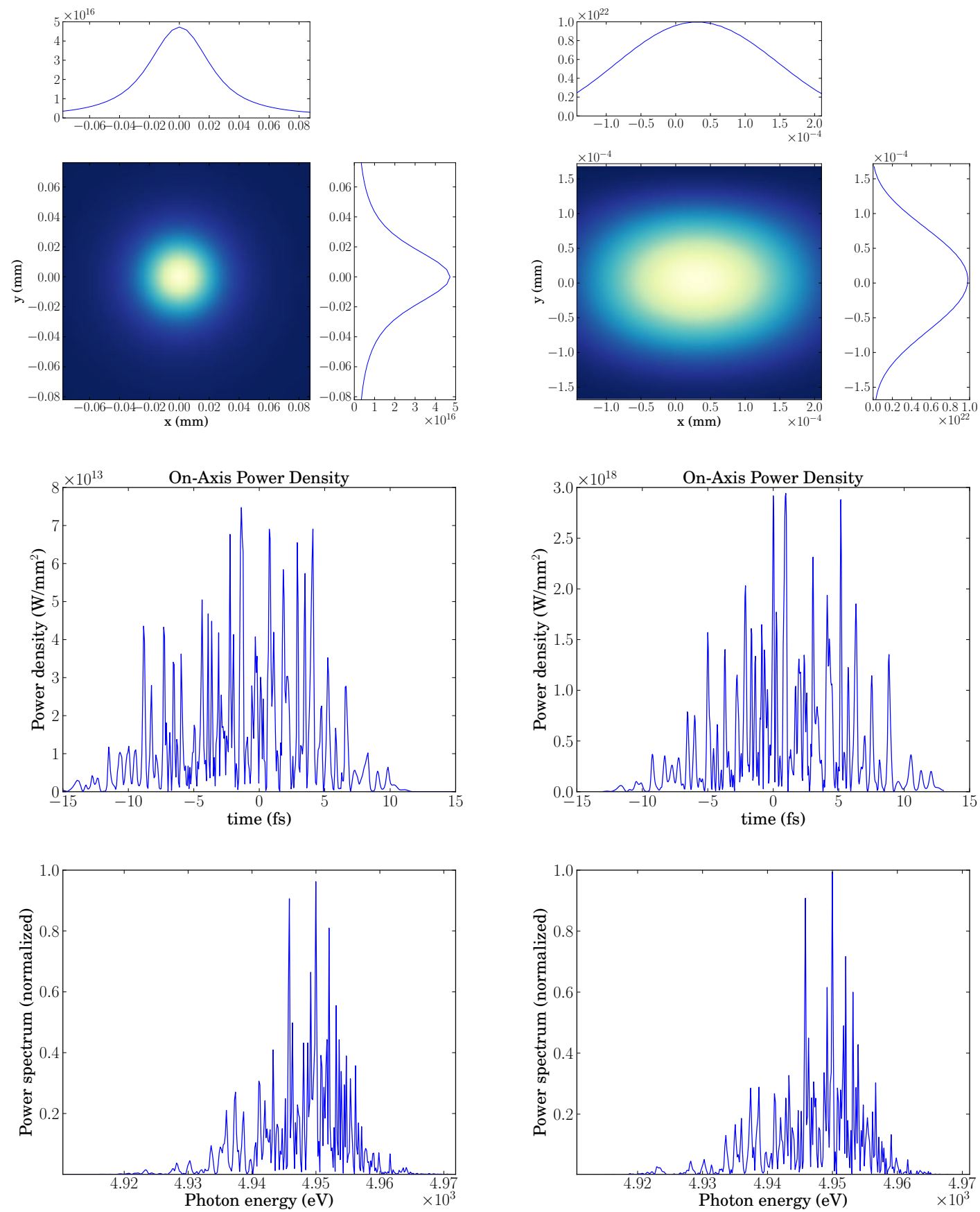

Fig 3 X-ray beam cross-section (top) on-axis power density (middle), and spectrum (bottom) at the source (left) and after propagation to focus (right).

the atomic positions as a function of time, the ionization state of each atom, and the atomic form factors and structure factors describing elastic and inelastic $\mathrm{x}$-ray scattering. It is also possible in simex_platform to switch off radiation damage completely, i.e. to perform the scattering 


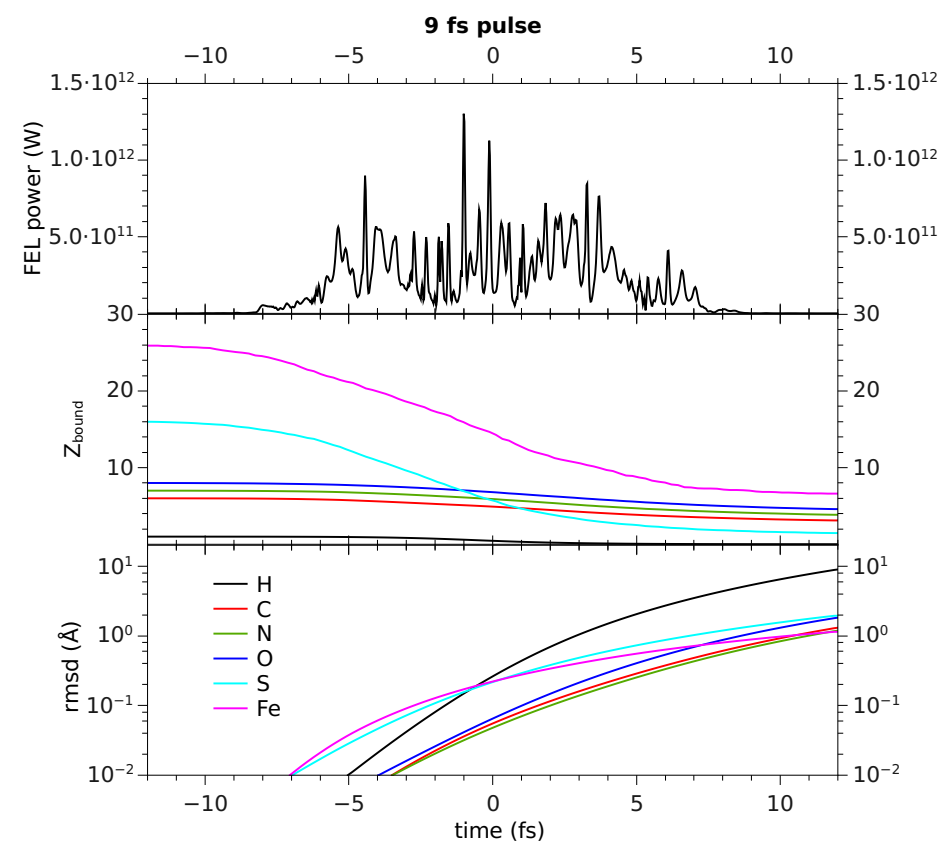

Fig $4 \mathrm{X}$-ray laser power, average ionization, and root-mean-square displacement (rmsd) as function of time for a 9 fs FWHM x-ray pulse at $5 \mathrm{keV}$ photon energy interacting with the 2NIP protein simulated with XMDYN and XATOM.

calculation based on the initial atomic positions and neutral ground state atomic form factors. This feature is very useful for quick test calculations, i.e. to get an estimate of detector signals and for debugging purposes. A comparison between simulated diffraction patterns with and without radiation damage will be given further below.

\subsection{Coherent $x$-ray diffraction}

The diffraction simulation code singFEL combines the outcome of the $\mathrm{x}$-ray beamline propagation and the simulation of photon-matter interaction and calculates for each time step the scattered intensity at a given pixel position as

$$
I(\mathbf{q})=\Omega \frac{d \sigma_{\mathrm{Th}}(\theta)}{d \Omega} \sum_{i} I_{0}\left(t_{i}\right)\left(\left|F\left(\mathbf{q}, t_{i}\right)\right|^{2}+S\left(\mathbf{q}, t_{i}\right)+N\left(t_{i}\right)\right) \Delta t
$$


where $q$ is the scattering wavevector given by the distance of the assumed pixel area detector from the sample and the size and position of the pixel for which we evaluate the scattered intensity; $\Omega$ is the solid angle spanned by the respective detector pixel, $d \sigma_{\mathrm{Th}} / d \Omega$ is the differential Thomson cross section, $I_{0}(t)$ is the FEL intensity at the sample position as function of time, which is the result of the $\mathrm{x}$-ray beamline propagation. $F(\mathbf{q}, t)$ is the bound electron form factor for coherent scattering while and $S(\mathbf{q}, t)$ and $N(\mathbf{q}, t)$ denote the incoherent contributions from bound and free electrons, respectively. The latter are taken from the photon-matter interaction calculation. The time stamp of the $i$ th snapshot is denoted $t_{i}$ and $\Delta t$ the time step.

To compose the diffraction pattern, the scattered intensities from each time step are incoherently added. Fig. 5 (top row) shows simulated diffraction intensities (on a log scale) as seen by a modelled pixel array located $13 \mathrm{~cm}$ downstream from the sample position. While the left graph was obtained from a calculation without radiation damage, i.e. using the initial atom positions and ground state atomic form factors in Eq. (1), the right viewgraph was obtained with radiation damage taken into account. In these simulations, the sample is the 2-Nitrogenase Iron Protein $(2 \mathrm{NIP}),{ }^{29}$ probed with a $0.5 \mathrm{~mJ} \mathrm{x}$-ray pulse at $4.96 \mathrm{keV}(0.25 \mathrm{~nm})$ and $9 \mathrm{fs}$ duration. As seen by comparison, radiation damage deteriorates the speckle contrast and overall scattering intensity. A more detailled discussion and comparison of results for different $\mathrm{x}$-ray pulse durations are given in the literature. ${ }^{6,30}$

\subsection{Detector response}

The detector is modelled as a $n_{x} \times n_{y}$ array of pixels of size $\sin$ the $x-y$ plane perpendicular to the $\mathrm{x}$-ray beam axis, intersecting the beam at a distance $d$ from the sample position.

From the scattered intensity, a Poissonized photon number is calculated for each pixel (indexed 

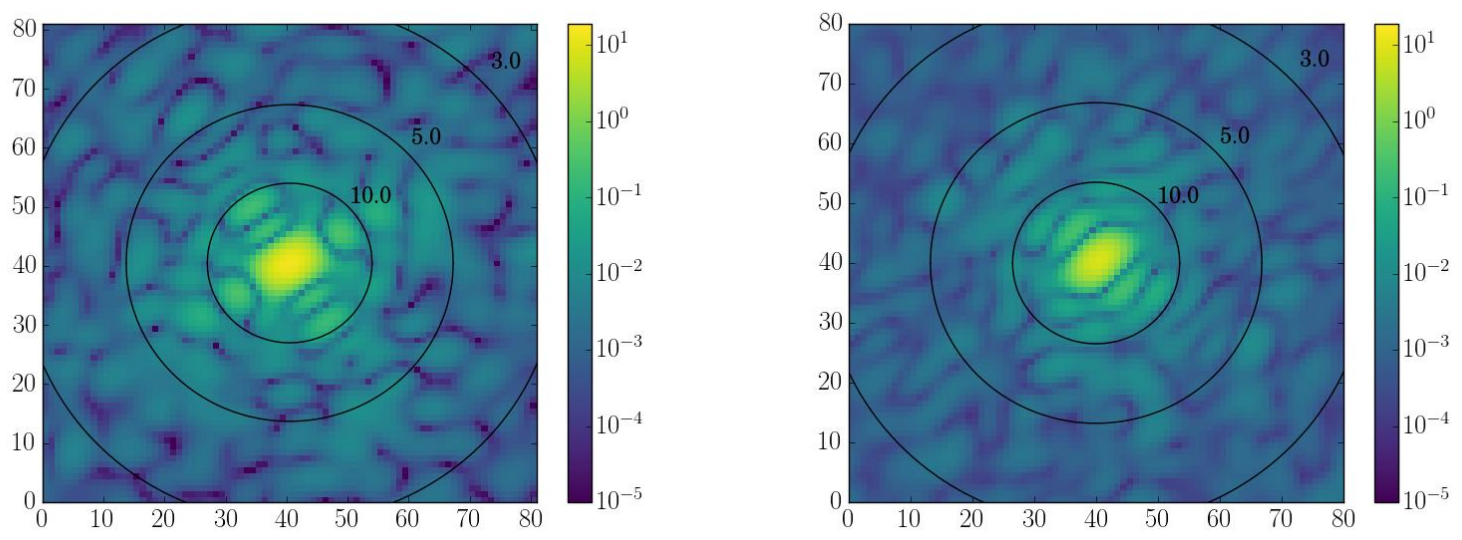

Fig 5 Simulated diffraction intensities without (left) and with (right) radiation damage. The sample is the 2NIP protein, photon energy $4.96 \mathrm{keV}$. The detector, an array of $80 \times 80$ pixels of sidelength $1.2 \mathrm{~mm}$ is placed $13 \mathrm{~cm}$ downstream from the sample. The circles mark the half-period resolution in $\AA$.

$j$ ): We first calculate an average photon number $\nu_{j}=I\left(q_{j}\right) s^{2} / E_{0}$, where $E_{0}$ is the initial photon energy of the x-ray laser. We then draw the detected photon count $n_{j}$ from a Poisson distribution around the average $P_{\nu_{j}}\left(n_{j}\right)=\nu_{j}^{n_{j}} \mathrm{e}^{-\nu_{j}} / n_{j}$ !. Fig. 6 shows the discretized pattern of detected photon numbers obtained after "Poissonization" of the corresponding intensity distribution shown in Fig. 5.
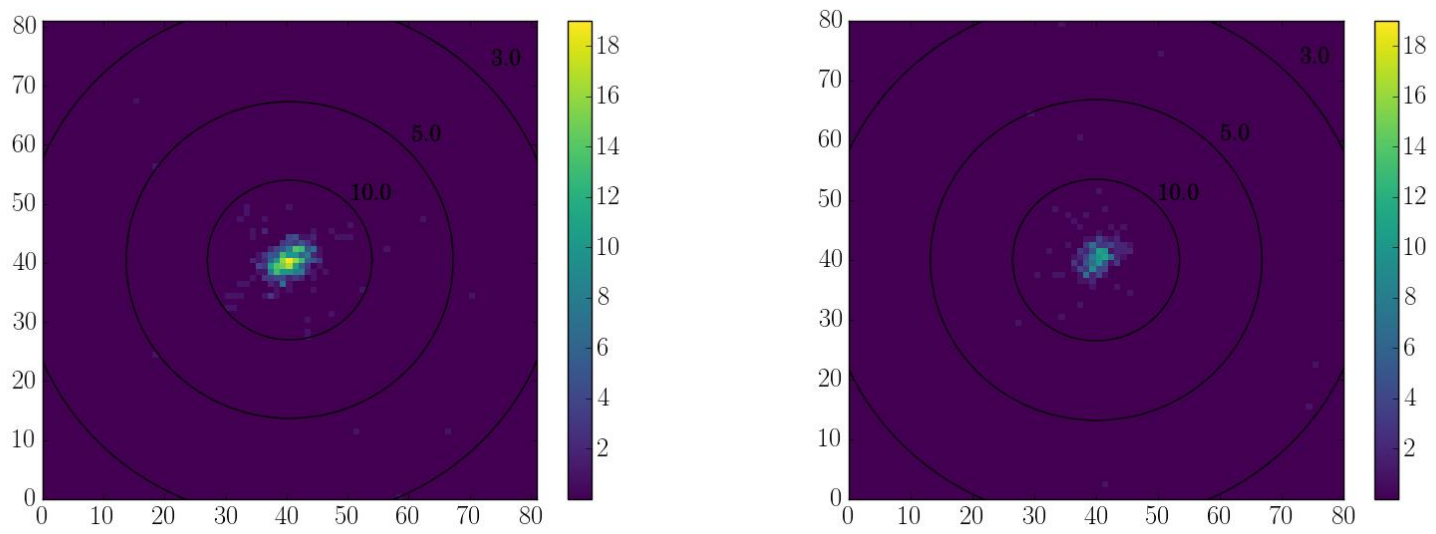

Fig 6 "Poissonized" photon counts corresponding to the diffraction intensities in Fig. 5.

More detailled simulations of the detector response to the incoming radiation can be performed 
using e.g. the $\mathrm{x}$-ray detector simulation toolkit $(\mathrm{X}--\mathrm{CSIT}),{ }^{31}$ which describes the electron creation mechanism and charge transport. An extension to $\mathrm{X}--\mathrm{CSIT}^{32}$ also simulates the detector electronics. In this way, various sources of detector noise, false positives and false negatives, dead pixels, hot spots, counting statistics and electronic noise can be taken into account. Integration of the extended X--CSIT software into simex_plat form is currently underway.

\subsection{Data analysis: Orientation and phasing}

Reconstruction of electron density maps from measured 2D diffraction patterns involves sophisticated data postprocessing steps: In a first step, all patterns must be re-oriented, i.e. the orientation of the sample molecule with respect to the $\mathrm{x}$-ray beam axis must be determined. Here, we employ the Expand-Maximize-Compress (EMC) algorithm. ${ }^{14,33}$ EMC has become the standard orientation algorithm for single-particle imaging at XFELs.

After orientation, the electron density can be reconstructed from the 3D diffraction volume using iterative phasing algorithms. ${ }^{15}$ Here, we use the Difference-Map algorithm as described e.g. in. ${ }^{14}$ Both EMC and DM are implemented in the emc_and_dm package. ${ }^{34}$

Besides these postprocessing algorithms, SIMEX also provides numerous utilities for the analysis of intermediate simulation results. We support statistical analysis and visualization of propagated radiation fields, photon-matter interaction results, diffraction patterns, an 3D volumetric data.

\section{X-ray Imaging of high power laser excited overdense plasmas}

Ultra-short pulsed high power lasers (HPLs) typically deliver laser pulses in the infrared (800 nm to $1000 \mathrm{~nm}$ ) at pulse durations below one picosecond. Today's HPLs ${ }^{35}$ can reach intensities of up 
to $10^{21} \mathrm{~W} \mathrm{~cm}^{-2}$ on spot sizes of a few microns and pulse durations on the order of a few tens of femtoseconds. Despite their intensity, these sources usually do not penetrate a solid density target but rather create a plasma at the target front side, accelerating electrons to relativistic energies in the strong electric field of the laser ${ }^{36}$ and pushing them into the target via the $\vec{v} \times \vec{B}$ force once the velocity $v$ approaches the speed of light. ${ }^{37,38}$

The generation of relativistic electrons at the front side, the transport of electrons through the target, and the subsequent formation of a sheath of electrons at the target rear side all happen on time scales below a few hundred femtoseconds. ${ }^{39}$ They can create plasma instabilities, ${ }^{40}$ ionize and heat the target bulk, ${ }^{41}$ generate strong magnetic fields, or drive shocks inside the target. These phenomena can potentially be studied with high spatial resolution of a few nanometers and temporal resolutions of a few femtoseconds using $\mathrm{x}$-ray lasers ${ }^{42,43}$ such as the European X-Ray Free Electron Laser (XFEL).

The Particle In Cell (PIC) method ${ }^{44}$ is an advanced simulation method to study the interaction of a HPL with a solid density target. Realistic 3D PIC simulations of laser-irradiated solid density plasmas require Petascale computing capabilities and produce hundreds of Terabytes of data. ${ }^{45}$

Within SIMEX we interface PIC codes such as PICOnGP $\mathrm{U}^{46,47}$ that describe the solid density plasma to codes that model the generation and propagation of XFEL pulses to generate synthetic scattering signals from free and, in the future, bound electrons.

As a first test of the simulation capabilities of simex_platform free electron density data from a PICONGPU simulation of the interaction of a short-pulse laser system (see details below) available at the High Energy Density (HED) instrument ${ }^{48}$ at the European XFEL will be used to compute a synthetic scattering signal in a Small-Angle X-ray Scattering (SAXS) geometry. 


\subsection{Experimental scenario}

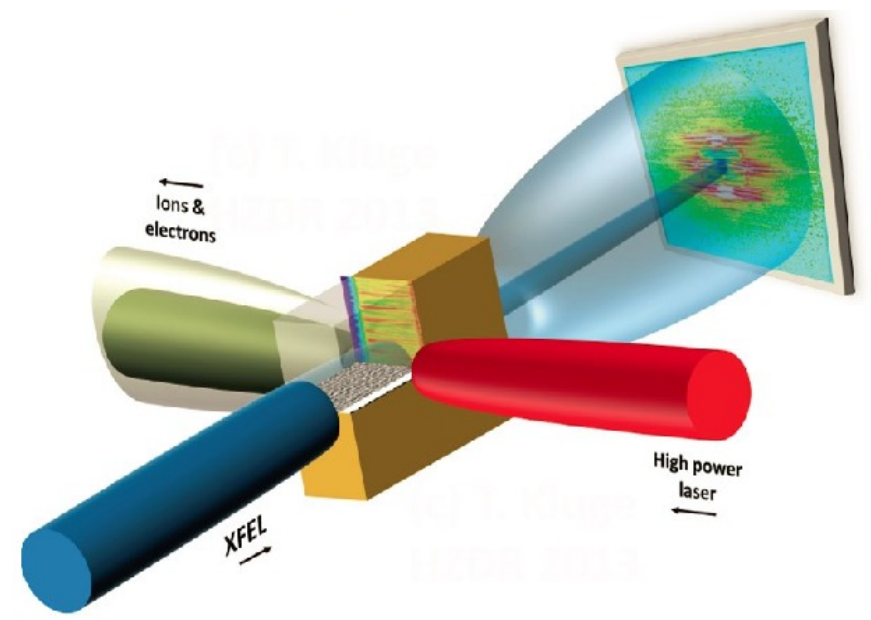

Fig 7 Schematic rendering of the proposed experiment to be simulated. Ultra-short pulsed optical laser radiation (red beam) creates a plasma layer on a metal foil's surface and accelerates electrons and ions. The XFEL beam (blue) probes the plasma perpendicular to the optical laser axis. A pixel area detector captures photons scattered in forward direction.
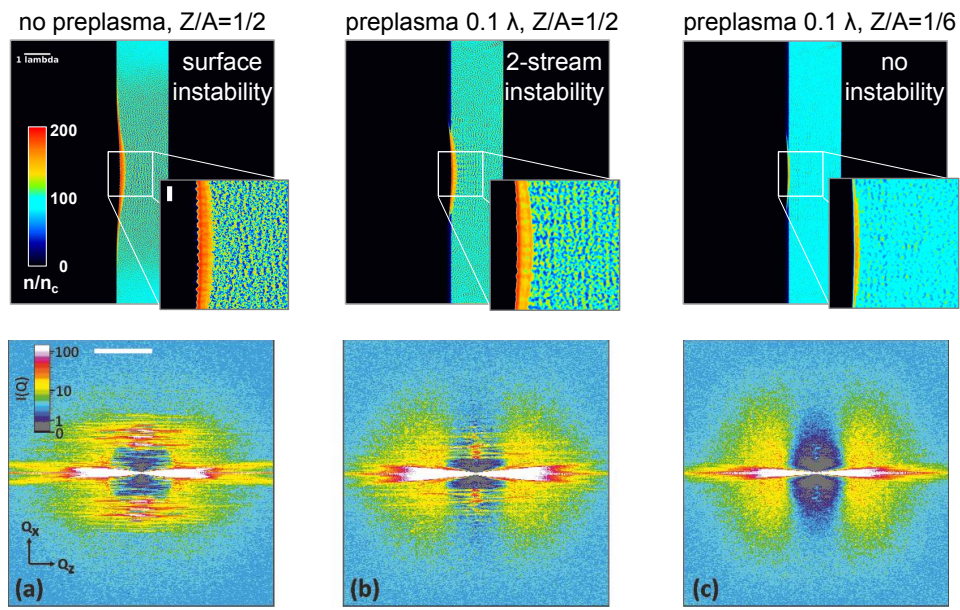

Fig 8 PIC simulations of the electron density (top) and forward scattering patterns (bottom) for various types of laser-plasma instabilities.

We briefly outline an experiment that we plan to simulate with the tools described above. The experiment would be carried out at the HED instrument at the European XFEL. X-ray parameters 
Table 1 X-ray pulse parameters for the HED instrument at the European XFEL. ${ }^{48,49}$

\begin{tabular}{|l|l|}
\hline Property & Range \\
\hline \hline photon energy (SASE mode) & $3 \mathrm{keV}$ to $25 \mathrm{keV}$ \\
photon energy (seeded) & $6 \mathrm{keV}$ to $12 \mathrm{keV}$ \\
\hline pulse duration & $1 \mathrm{fs}$ to $100 \mathrm{fs}$ \\
\hline focus size (FWHM) & $<1 \mu \mathrm{m}, 1 \mu \mathrm{m}, 15 \mu \mathrm{m}$ and $100 \mu \mathrm{m}$ \\
\hline rel. bandwidth & $10^{-3}(\mathrm{SASE})$ to $10^{-5}$ (seeded) \\
\hline photons/pulse & $10^{12}$ to $10^{13}$ \\
\hline
\end{tabular}

Table 2 European XFEL HED PP laser parameters for optical laser-matter interaction simulations ${ }^{48,49}$

\begin{tabular}{|l|l|}
\hline Property & Range \\
\hline \hline wavelength & $800 \mathrm{~nm}$ \\
\hline pulse duration (FWHM) & $15 \mathrm{fs}$ to $300 \mathrm{fs}$ \\
\hline focus size (FWHM) & $3 \mu \mathrm{m}$ \\
\hline temporal profile & Gaussian \\
\hline spatial profile & Gaussian \\
\hline pulse energy & $2 \mathrm{~mJ}$ \\
\hline prepulse energy contrast & $\approx 10^{-4}$ \\
\hline intensity & $10^{17} \mathrm{~W} \mathrm{~cm}^{-2}$ \\
\hline
\end{tabular}

are given in Tab. 1. We propose a SAXS setup, illustrated in Fig. 7, where a thin metal foil is first excited using the short pulse HED PP laser (parameter are listed in Tab. 2 and subsequently probed by XFEL photons. Other laser systems available at the HED instrument, including more powerful (of the order $100 \mathrm{TW}$ ) lasers are described, in Ref. ${ }^{49}$

The choice of the target material depends on the precise $\mathrm{x}$-ray photon energy. The target material's K-shell absorption edge should be located slightly below the probe's wavelength to limit $\mathrm{x}$-ray absorption. In resonant SAXS, the target material's K-shell transitions must match the $\mathrm{x}$-ray photon energy. Assuming a photon energy of $8 \mathrm{keV}$, copper is a suitable target material for normal SAXS and resonant SAXS.

The HED PP laser will create a plasma of a few to ten micrometer size in the transverse dimension with respect to the XFEL beam. Hence, we choose $10 \mu \mathrm{m}$ for the focal spot size to irradiate 
the full plasma length. A relative bandwidth of $\approx 10^{-3}$ in SASE mode, corresponding to a few eV in absolute energy is suitable for a resonant SAXS experiment to ensure efficient excitation of all involved transitions. This bandwidth is also adequate for non-resonant SAXS.

Through observation of distinct features in the scattering signal being signatures of resonant modes and instabilities in the plasma, we will extract information about these modes such as their amplitude and growth rate under varying experimental conditions such as laser power, incidence and probe angles. Fig. 8 shows three simulated scattering patterns emerging from interaction of coherent $\mathrm{x}$-rays with different types of plasma instabilities. ${ }^{42}$ It should be noted that these simulations make idealistic assumptions about the properties of the x-ray probe. An important aspect of this work is the question if the observed characteristics will remain so clearly distinct if realistic simulated pulses are employed in the scattering simulation. Comparison to experimental data will serve as a validation check of the simulation chain.

\subsection{Simulation workflow}

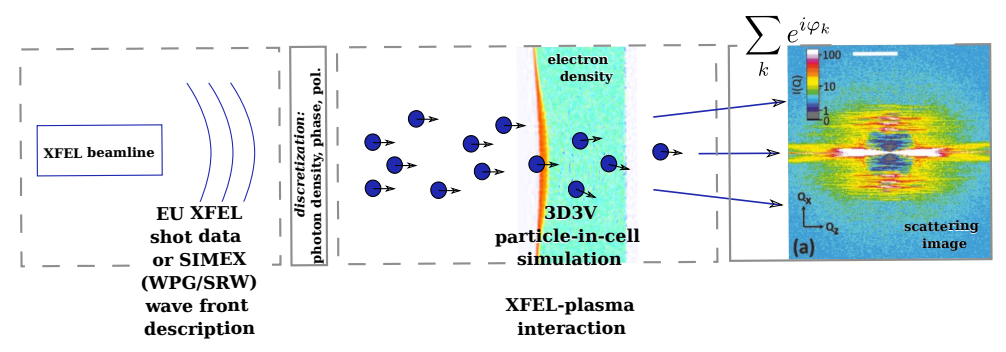

Fig 9 Block diagram for integrated simulations of $\mathrm{x}$-ray scattering from a high power laser excited plasma.

The simulation workflow is depicted in Fig. 9. The $\mathrm{x}$-ray pulse generation and propagation is modeled in simex_platform with the corresponding simulation codes. The simulated wavefronts are evaluated at the target surface and converted into a photon distribution. The photons are then propagated through the target. At discrete timesteps, each photon's wavevector undergoes 
a change in magnitude and direction with a certain probability, given by the Thomson scattering cross section. ${ }^{50}$ In this way, multiple scattering events that become important especially in compressed regions of the target, are naturally included. Finally, the photon wavefunction is collapsed onto the detector plane and integrated over the exposure time to obtain the simulated scattering image.

\subsection{Propagation}

Propagation of XFEL pulses is modeled through the XFELPhotonPropagator. The simulation parameters reflect the conditions at the High Energy Density (HED) instrument ${ }^{48}$ at the European XFEL. The focal spotsize can be fixed in three different ranges around $2 \mu \mathrm{m}, 20 \mu \mathrm{m}$ and $200 \mu \mathrm{m}$ FWHM.

\subsection{Photon-Matter interaction}

\subsubsection{Optical Laser}

The short-pulse optical laser-plasma interaction is modeled with P IConGPU. ${ }^{46}$ P IConGPU is an Open Source ${ }^{47}$ explicit, relativistic $3 \mathrm{D} 3 \mathrm{~V}^{1}$ PIC code which can simulate the interaction of high power, ultra-short laser pulses with matter. The simulations will assume realistic parameters for the optical laser system at the HED instrument at European XFEL. The most important parameters are summarized in Table 2. HPL pulses are defined by their time dependent magnetic and electric field components. Their evolution is governed by the Maxwell equations. ${ }^{50}$ A PIC code solves these partial differential equations on a regular mesh (usually Cartesian) using e.g. finite difference time domain techniques. ${ }^{51}$

\footnotetext{
${ }^{1} 3 \mathrm{D} 3 \mathrm{~V}$ denotes the six dimensional phase space spanned by three position vector components and three velocity vector components
} 


\subsubsection{X-ray Laser (Scattering)}

In principle, all imaging techniques employed for probing the interaction of HPL lasers with targets can be used in the case of ultra-short pulse lasers as well. However, some of these techniques are limited to slowly varying plasma conditions (in comparison to the ultra-short laser pulse duration) and thus are not suitable to capture fast, transient processes. Moreover, atomic processes, multiple scattering, velocity-dependent scattering and non-equilibrium plasma conditions cannot always be taken into account with existing modeling techniques, especially if the x-ray laser pulse itself interacts with the plasma.

Thus, for ultra-short laser pulses $\mathrm{x}$-ray interaction will in addition to existing techniques be modeled via a Monte-Carlo photon interaction model. Each photon is described by its wave vector and a phase ${ }^{2}$.

The $\mathrm{x}$-ray pulse is described by temporally and spatially varying electric fields (amplitude, polarization, and phase). At discrete points in time, the field distribution in a plane perpendicular to the laser axis is converted into a photon distribution using a conversion tool contained in simex_platform. These photons are then tracked through the volume simulated by P IConGPU using the software parataxis. Table 1 gives an overview over the expected $\mathrm{x}$-ray pulse parameters at the HED instrument at the European XFEL. In describing the interaction of coherent x-rays with the laser excited plasma, we focus on scattering processes, predominantly Thomson scattering from free electrons. Further atomic processes, e.g. K-shell ionization and resonant scattering will be considered later when detailed atomic modelling using the scFLY code ${ }^{52}$ has become part of the simulation capabilities. This is currently under development.

The scattering signal will be processed to infer information about the microscopic and macro-

\footnotetext{
${ }^{2}$ In the future, the photon model will also include polarization.
} 
scopic state of the plasma during and after the optical laser-plasma interaction. Photons are scattered according to predefined scattering functions that depend on the local properties of the matter irradiated by the HPL. In the most simple case, the local free electron density is used as an input for calculating the probability for Thomson scattering from free electrons.

In the context of PIC simulations, several methods for scattering calculations are or will be implemented. Ex-situ techniques act as a post-processor on PIC data, e.g. using Fast Fourier Transforms $^{53}$ or Monte-Carlo tracking of photons through the simulation volume. The latter is implemented in the code paraTAXIS. In contrast, in-situ techniques calculate the scattering while the PIC simulation is running using e.g. Lienard-Wiechert potentials ${ }^{54}$ or by adding photons as a new particle species to the PIC simulation.

Fig. 10 shows PIC simulation data (left panel) and an ex-situ simulated SAXS image (right panel) from a solid hydrogen target irradiated by $800 \mathrm{~nm}$ short-pulse (30 fs) laser radiation and imaged by a $1 \AA$ wavelength $\mathrm{x}$-ray pulse. Details of the calculation and experimental parameters are given in the caption, see also Ref. ${ }^{57}$

In addition to elastic scattering of photons from electrons, excitation of ions, absorption, emission, and Compton scattering will be considered in the future. The latter are incoherent processes contributing to the radiation background and their consideration is hence an important step towards a more realistic simulation of the scattering signal.

\section{Warm dense matter production through high energy laser shock compression and $x-$ ray radiography diagnostics}

High-energy laser facilities are now becoming increasingly in demand at new generation $\mathrm{x}$-ray light sources (X-FELs, 3rd generation synchrotron sources). The potential of long pulse ( $\approx 0.1 \mathrm{~ns}$ 

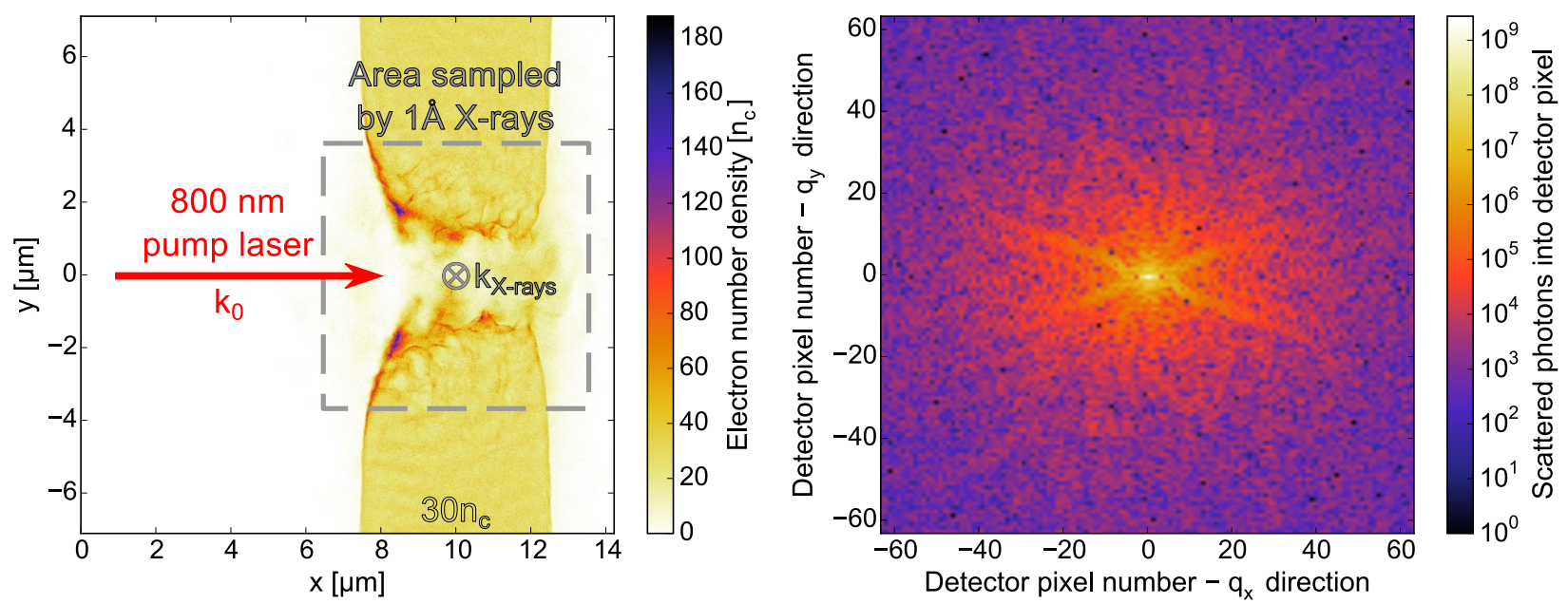

Fig 10 SAXS signal from a laser-driven cryogenic hydrogen strand. The left image shows the electron number density distribution $134 \mathrm{fs}$ after a $30 \mathrm{fs}, 105 \mathrm{TW}$ infrared driver laser pulse has hit the $5 \mu \mathrm{m}$ thick solid hydrogen strand. The laser is incident from the left and focused to a spot size of $3 \mu \mathrm{m}$ full-width at half maximum of the intensity profile. The initial hydrogen number density is thirty critical densities $n_{\mathrm{c}}=\epsilon_{0} m c^{2} k_{0}^{2} / q^{2}$ for the laser wavelength of $\lambda_{\text {Laser }}=800 \mathrm{~nm}$. During laser irradiation electrons are pushed by the laser ponderomotive force leading to hole boring and an increase in density near the laser propagation axis. The electron density distribution can be imaged by SAXS with an $\mathrm{x}$-ray pulse of femtosecond scale duration. X-ray pulse and pump laser direction of propagation are perpendicular such that the $1 \AA$ wavelength $\mathrm{x}$-ray pulse illuminates the target from the side on a $7 \times 7 \mu \mathrm{m}^{2}$ area. The right image shows a region of interest from the corresponding synthetic scattering signal on an $\mathrm{x}$-ray camera $93 \mathrm{~cm}$ behind the target. It is obtained by a Fourier transform of the two dimensional electron density data of the area illuminated by the XFEL pulse assuming that all free electrons of the $5 \mu \mathrm{m}$ thick, fully ionized target contribute to the scattering signal and that the density does not change siginificantly during XFEL pulse illumination. The incident $\mathrm{x}-$ ray pulse parameters match those expected for the European XFEL HED instrument ${ }^{49,55}$ which provides $10^{12}$ photons. With photon counts in the range of $10^{5}$ to $10^{6}$ per $13 \times 13 \mu \mathrm{m}^{2}$ detector pixel the scattering signal is well-measurable within a single shot which proves the feasibility to record femtosecond and nanometer scale dynamics of laser-driven plasmas at XFELs. Electron density data was obtained by a PICLS ${ }^{56}$ simulation.

to $10 \mathrm{~ns}$ ) laser experiments are already yielding extreme conditions of matter well beyond the reach of static high-pressure techniques and combinations with $\mathrm{x}$-ray techniques provide enticing experiments at new extreme states of matter.

Temporally shaped laser pulses interacting with overdense target material create a rapidly expanding plasma that can generate a shockwave in the ablating material. This shock wave can travel through the target at several $\mathrm{km} / \mathrm{s}$ and compress the sample material to pressures reaching several hundred GPa. (For comparison, the Earth's core pressure is $\approx 330 \mathrm{GPa}$ ). By using a ramp temporal pulse, where the laser intensity is slowly increased, the temperatures generated remain much cooler 
than during rapid shock compression and the solid state of matter can be studied up to several TPa $(1 \mathrm{TPa}=1000 \mathrm{GPa})$

Long pulse laser systems are capable of reaching intensities of up to $\approx 10^{12} \mathrm{~W} \mathrm{~cm}^{-2}$ to $10^{15} \mathrm{~W} \mathrm{~cm}^{-2}$ with pulse lengths of greater than a few nanoseconds. The pulse length and focal spot of the laser can depend heavily on the sample material and target package. Generally, focal spot sizes range between $\approx 0.1 \mathrm{~mm}$ to $1 \mathrm{~mm}$. With these specifications, the laser energies now required to reach the highest intensities are on the order of hundreds of joules. Table 3 summarizes the main optical pump laser parameters for shock compression experiments at the ESRF.

Table 3 ESRF optical laser parameters for long pulse laser-matter simulations.

\begin{tabular}{|l|l|}
\hline Property & Range \\
\hline Wavelength & $1053 \mathrm{~nm}$ \\
\hline Pulse duration & $4 \mathrm{~ns}$ to $15 \mathrm{~ns}$ \\
\hline Intensity & $2 \times 10^{13} \mathrm{~W} \mathrm{~cm}^{-2}$ \\
\hline Pulse energy & 30 joule \\
\hline Focus size (fhwm) & $100 \mu \mathrm{m}^{2}$ to $300 \mu \mathrm{m}^{2}$ \\
\hline Temporal profile & Quasi-flat top \\
\hline Spatial profile & Gaussian in $\mathrm{x}, \mathrm{y}$ \\
\hline
\end{tabular}

A new high-energy laser system to with increased energy (200 J and frequency doubling crystals will shortly be installed at the ESRF. A similar experiment could also be executed at European XFEL using the long pulse laser system of the HED instrument. Here, owing to the orders of magnitude higher photon fluences, the system could also be probed by inelastic $\mathrm{x}$-ray scattering. ${ }^{58}$

We design here simulations for a prototypical experiment combining a high-energy laser system and $\mathrm{x}$-ray radiation from a 3rd generation synchrotron. The laser shock-compresses a tailored solid density target and the compressed matter is subsequently probed by $\mathrm{x}$-rays. X-ray absorption spectroscopy (XAS) allows to monitor the compression and to characterize the electronic and 
Table 4 Principal $\mathrm{x}$-ray parameters for shock compression studies at ESRF (beamline ID24)

\begin{tabular}{|l|l|}
\hline Property & Range \\
\hline Photon energy & $5 \mathrm{keV}$ to $28 \mathrm{keV}$ \\
\hline Polarization & horizontal \\
\hline Focus size (FWHM) & $3 \mu \mathrm{m}(5 \mathrm{keV})$ to $50 \mu \mathrm{m}(28 \mathrm{keV})$ \\
\hline Pulse length & $100 \mathrm{ps}$ \\
\hline Photon flux & $10^{14} \mathrm{~s}^{-1}(7 \mathrm{keV})$ to $4 \times 10^{13} \mathrm{~s}^{-1}(28 \mathrm{keV})$ \\
\hline
\end{tabular}

structural states in the target. Through variation of the delay between optical laser pulse and $\mathrm{x}-$ ray probe pulse, time resolved data can also obtained.

\subsection{Experimental scenario}

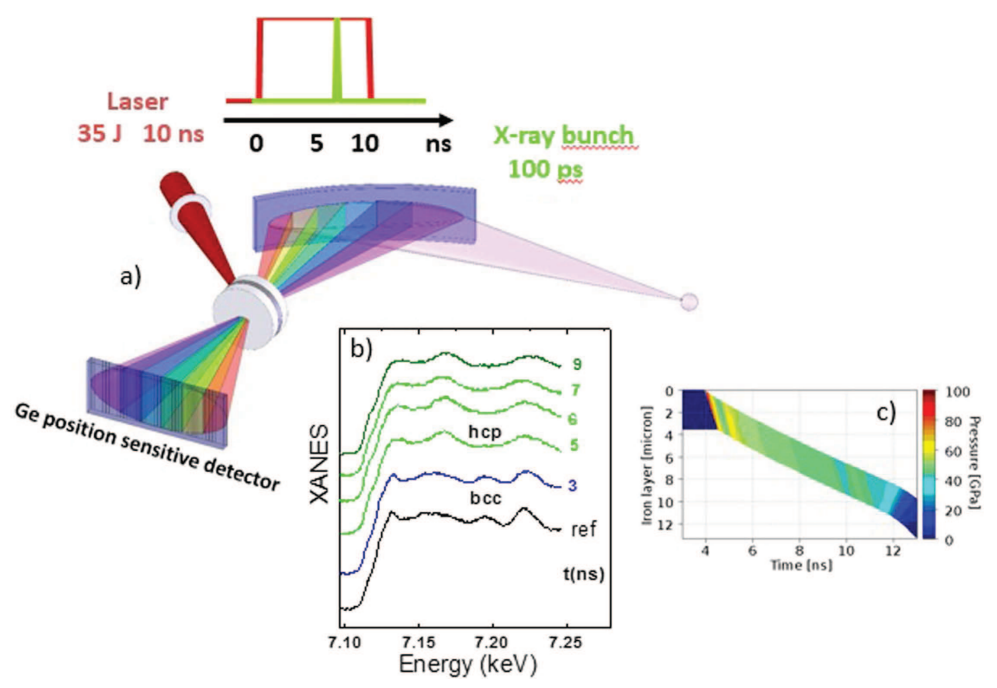

Fig 11 Schematic rendering of a laser driven shock compression experiment and $\mathrm{x}$-ray absorption spectroscopy (a). XANES signal as function of time indicating structural phase transition from bcc to hcp phase (b). 1-D Radiationhydrodynamics simulation of the shock propagation and pressure dynamics during laser-matter interaction (c). Reproduced from Ref..$^{59}$

Fig. 11 shows the experimental setup, optical laser and $\mathrm{x}$-ray probe pulse timings (a), measured XANES spectra (b) and 1D radiation-hydrodynamic simulations of shock propagation through the a sandwiched iron target. The figure is taken from Ref. ${ }^{59}$ The drive pulse compresses the target as modeled by radiation hydrodynamics simulations reaching pressures in the range of $40 \mathrm{GPa}$ to 
$60 \mathrm{GPa}$. The $\mathrm{x}$-ray pulse of $\approx 100$ ps probes the compressed target after a variable time delay. $\mathrm{X}$-ray pulses for shock compression studies at the ESRF are $\approx 100 \mathrm{ps}$ long. Table 4 lists the most important $\mathrm{x}$-ray parameters. Transmitted $\mathrm{x}$-ray photons are detected in a position sensitive $\mathrm{Ge}$ detector. The position of the detected photon can be converted into photon energy employing the dispersion curve of the focussing mirror.

We simulate this experiment using the available tools in simex_platform. In addition, simex_platform provides tools for iterative optimization of the optical laser pulse parameters and the target geometry (ablator thickness and material, target thickness, window material. Details about the simulation Backengines are provided in the following.

\subsection{Photon-Matter interaction}

\subsubsection{Optical laser and radiation-hydrodynamic codes}

Nearly all of the physical processes of long pulse laser-matter interactions are described by partial differential equations that can be solved with careful construction of numerical simulation codes. Here, the laser-matter interactions are modelled with the 1D radiation-hydrodynamics computer code ESTHER ${ }^{60}$ or the 2D code MULTI2D. ${ }^{61}$ For the $1 \mathrm{D}$ hydrocodes, a moving mesh is applied in an arbitrary Lagrangian-Eulerian framework where the coordinates of the mesh contain the variables from which density can be calculated (mass of each zone is fixed). The most important variables considered in these codes are those associated with the extreme conditions generated by the high-power lasers: pressure (density), temperature and velocity. Feedback of these hydrocode outputs are crucial in the design and implementation of laser shock/ramp experiments with $\mathrm{x}$-ray interactions.

It is vital to have an understanding of shock transit times so that accurate timing of the $\mathrm{x}$-ray 
probe, with respect to the laser initialisation, can be made. The final density state reached by a shock, as calculated from the hydrocode simulation packages, can define the shock velocity.

\section{$4.3 X$-ray matter interaction}

In the proposed experimental setup, the shock compressed matter is diagnosed by X-ray Absorption Fine Structure (XAFS) spectroscopy and radiography. The XAFS signal reflects the near order of the shock compressed matter. The absorption edge is modulated by the ion-ion correlation function $\chi(\vec{q})$ making it possible to identify the crystalline structure in the absorption spectrum. XAFS can be simulated by combining first principle electronic structure methods with linear response theory. A well known implementation is the code FEFF. ${ }^{62}$

Radiography can be used to image e.g. the shock front during dynamical compression. The raytracing software ShadowOUI ${ }^{63}$ has the capability to calculate radiographs from simulated density profiles.

\section{Conclusions}

In summary, we have outlined the capabilities of our generic photon science simulation platform simex_platform. It provides user interfaces for and data interfaces between advanced simulation codes for simulations of the complete experiment for at least three types of applications: SPI experiments at XFELs, $\mathrm{x}$-ray probing of short-pulse laser excited matter and $\mathrm{x}$-ray diagnostics of dynamically compressed matter. Starting from the source, photons are propagated to the sample or target by means of $\mathrm{x}$-ray tracing or coherent wavefront propagation. Interaction with the sample can be simulated using MD for finite systems (biomolecules, clusters), PIC and ex-situ Monte Carlo photon tracking for the femtosecond dynamics of matter after excitation by short- 
pulse laser irradiation, and radiation-hydrodynamics coupled to electronic structure calculations for long-pulse laser compression of solid matter and subsequent $\mathrm{x}$-ray probing. Our platform is available under the GNU General Public License (version 3). ${ }^{64}$ It can be installed from the sources, via binary packages or as a docker ${ }^{65}$ container.

Besides accomodation of further physics backengines and execution of the applications discussed in this paper, we will also extend the platform to allow more complex simulations. As an example, we mention simulations of a compact coherent light source based on relativistic laserwakefield acceleration coupled to an undulator beamline simulator.

\section{Disclosures}

The authors declare no conflict of interests.

\section{Acknowledgments}

CFG, RB, MG, AG, AH, AS, and SY acknowledge support from the European Cluster of Advanced Laser Light Sources (EUCALL) project which has received funding from the European Unions Horizon 2020 research and innovation programme under grant agreement No 654220.

\section{References}

1 C. Fortmann-Grote, A. A. Andreev, R. Briggs, et al., "Simex: Simulation of experiments at advanced light sources," Journal of Physics Conference Series (accepted) (2016).

2 https://github.com/eucall-software/simex_platform.

3 G. van Rossum, "Python tutorial,” Tech. Rep. CS-R9526, Centrum voor Wiskunde en Informatica (CWI), Amsterdam, Netherlands (1995). 
4 Python Software Foundation, "Python Language Reference, version 2.7." http://www.python.org.

5 The HDF Group, "Hierarchical Data Format, version 5," (1997). http://www.hdfgroup.org/HDF5/.

6 C. H. Yoon, M. V. Yurkov, E. A. Schneidmiller, et al., "A comprehensive simulation framework for imaging single particles and biomolecules at the european x-ray free-electron laser.," Scientific reports 6, 24791 (2016).

7 https://sims2e.readthedocs.io/en/latest/.

8 A. Huebl, R. Lehe, J.-L. Vay, et al., “openPMD 1.0.0: Meta Format for Particle and Mesh Based Data," (2015). http://www.openpmd.org.

9 R. Neutze, R. Wouts, D. van der Spoel, et al., "Potential for biomolecular imaging with femtosecond x-ray pulses.," Nature 406, 752-7 (2000).

10 A. Barty, C. Caleman, A. Aquila, et al., "Self-terminating diffraction gates femtosecond x-ray nanocrystallography measurements," Nature Photonics 6, 35-40 (2011).

11 S. P. Hau-Riege, "Photoelectron dynamics in x-ray free-electron-laser diffractive imaging of biological samples," Physical Review Letters 108, 238101 (2012).

12 A. Aquila, A. Barty, C. Bostedt, et al., "The linac coherent light source single particle imaging road map," Structural Dynamics 2, 041701 (2015).

13 O. Y. Gorobtsov, U. Lorenz, N. M. Kabachnik, et al., "Theoretical study of electronic damage in single-particle imaging experiments at x-ray free-electron lasers for pulse durations from 0.1 to 10 fs," Physical Review E 91 (2015). 
14 N.-T. D. Loh and V. Elser, "Reconstruction algorithm for single-particle diffraction imaging experiments," Physical Review E 80, 026705 (2009).

15 J. R. Fienup, "Phase retrieval algorithms: a comparison," Appl. Opt. 21, 2758-2769 (1982).

16 E. Wolf, Introduction to the Theory of Coherence and Polarization of Light, Cambridge University Pr. (2007).

17 O. Chubar, M.-E. Couprie, M. Labat, et al., “Time-dependent fel wavefront propagation calculations: Fourier optics approach," Nuclear Instruments and Methods in Physics Research Section A: Accelerators, Spectrometers, Detectors and Associated Equipment 593, 30-34 (2008).

18 European XFEL GmbH, “XFEL Photon pulses Database XPD.” https://in.xfel.eu/xpd/.

19 E. L. Saldin, E. A. Schneidmiller, and M. V. Yurkov, "Fast: A three-dimensional timedependent fel simulation code," Nucl. Instrum. Methods Phys. Res., Sect. A 429(1), 233-237 (1999).

20 S. Reiche, “Genesis 1.3: a fully 3d time-dependent fel simulation code," Nuclear Instruments and Methods in Physics Research Section A: Accelerators, Spectrometers, Detectors and Associated Equipment 429, 243-248 (1999).

21 L. Samoylova, A. Buzmakov, O. Chubar, et al., "WavePropaGator: interactive framework for X-ray free-electron laser optics design and simulations," J Appl Cryst 49, 1347-1355 (2016).

22 O. Chubar, P. Elleaume, S. Kuznetsov, et al., "Physical optics computer code optimized for synchrotron radiation," in Proc. SPIE 4769, Optical Design and Analysis Software II, R. C. Juergens, Ed., 4769, 145-151 (2002). 
23 B. F. Murphy, T. Osipov, Z. Jurek, et al., "Femtosecond x-ray-induced explosion of c60 at extreme intensity," Nature Communications 5 (2014).

24 Z. Jurek, S.-K. Son, B. Ziaja, et al., "XMDYN and XATOM: versatile simulation tools for quantitative modeling of X-ray free-electron laser induced dynamics of matter,' Journal of Applied Crystallography 49, 1048-1056 (2016).

25 S.-K. Son, L. Young, and R. Santra, "Impact of hollow-atom formation on coherent x-ray scattering at high intensity," Phys. Rev. A 83(3), 33402 (2011).

26 B. Rudek, S.-K. Son, L. Foucar, et al., "Ultra-efficient ionization of heavy atoms by intense X-ray free-electron laser pulses," Nature Photonics 6, 858-865 (2012).

27 H. Fukuzawa, S.-K. Son, K. Motomura, et al., "Deep inner-shell multiphoton ionization by intense x-ray free-electron laser pulses,” Physical Review Letters 110 (2013).

28 T. Tachibana, Z. Jurek, H. Fukuzawa, et al., "Nanoplasma formation by high intensity hard x-rays," Sci. Rep. 5, 10977 (2015).

29 J. L. Schlessman, D. Woo, L. Joshua-Tor, et al., "Conformational variability in structures of the nitrogenase iron proteins from azotobacter vinelandii and clostridium pasteurianum," Journal of Molecular Biology 280, 669-685 (1998).

30 C. Fortmann-Grote et al., "Start-to-end simulation of single particle imaging using ultra-short pulses at the European X-ray Free Electron Laser,” (2017). submitted.

31 A. Joy, M. Wing, S. Hauf, et al., "X-csit: a toolkit for simulating 2d pixel detectors," Journal of Instrumentation 10, C04022-C04022 (2015).

32 T. Rüter, S. Hauf, M. Kuster, et al., "X-ray detector simulation pipelines for the european xfel," in IEEE Conference Proceedings of the Nuclear Science Symposium 2015, (2016). 
33 K. Ayyer, T.-Y. Lan, V. Elser, et al., "Dragonfly: an implementation of the expand-maximize-compress algorithm for single-particle imaging," J Appl Cryst 49, 1320_ 1335 (2016).

34 https://www.github.com/eucall-software/emc_and_dm.

35 M. Siebold, J. Hein, M. Hornung, et al., "Diode-pumped lasers for ultra-high peak power," Applied Physics B 90(3), 431-437 (2008).

36 T. Kluge, T. Cowan, A. Debus, et al., "Electron temperature scaling in laser interaction with solids," Phys. Rev. Lett. 107, 205003 (2011).

37 P. Mulser and D. Bauer, High Power Laser-Matter Interaction, Springer Tracts in Modern Physics, Springer Berlin Heidelberg (2010).

38 P. Gibbon and E. Förster, "Short-pulse laser - plasma interactions," Plasma Physics and Controlled Fusion 38(6), 769 (1996).

39 A. Macchi, M. Borghesi, and M. Passoni, "Ion acceleration by superintense laser-plasma interaction," Rev. Mod. Phys. 85, 751-793 (2013).

40 J. Metzkes, T. Kluge, K. Zeil, et al., "Experimental observation of transverse modulations in laser-driven proton beams," New Journal of Physics 16, 023008 (2014).

41 L. G. Huang, M. Bussmann, T. Kluge, et al., "Ion heating dynamics in solid buried layer targets irradiated by ultra-short intense laser pulses," Physics of Plasmas 20, 093109 (2013).

42 T. Kluge, C. Gutt, L. G. Huang, et al., "Using x-ray free-electron lasers for probing of complex interaction dynamics of ultra-intense lasers with solid matter," Physics of Plasmas 21, 033110 (2014). 
43 T. Kluge, M. Bussmann, H.-K. Chung, et al., "Nanoscale femtosecond imaging of transient hot solid density plasmas with elemental and charge state sensitivity using resonant coherent diffraction," Physics of Plasmas 23, 033103 (2016).

44 C. Birdsall and A. Langdon, Plasma Physics via Computer Simulation, Series in Plasma Physics, CRC Press (2004).

45 Oak Ridge National Laboratory, “"'Titan targets tumors".” https://www.ornl.gov/news/titantargets-tumors.

46 M. Bussmann, F. Schmitt, U. Schramm, et al., "Radiative signatures of the relativistic kelvinhelmholtz instability," in Proceedings of the International Conference for High Performance Computing, Networking, Storage and Analysis on - SC'13, SC '13, 1-12, ACM Press, (New York, New York, USA) (2013).

47 https://github.com/ComputationalRadiationPhysics/picongpu.

48 M. Nakatsutsumi, K. Appel, G. Priebe, et al., “Technical Design Report: Scientific Instrument High Energy Density Physics (HED),” tech. rep., European XFEL GmbH (2014).

49 M. Nakatsutsumi, K. Appel, C. Baehtz, et al., "Femtosecond laser-generated high-energydensity states studied by x-ray FELs," Plasma Physics and Controlled Fusion 59, 014028 (2016).

50 J. D. Jackson, “Classical electrodynamics,” (1975).

51 K. Yee, "Numerical solution of initial boundary value problems involving maxwell's equations in isotropic media," IEEE Transactions on Antennas and Propagation 14, 302-307 (1966). 
52 H.-K. Chung, M. Chen, and R. Lee, "Extension of atomic configuration sets of the non-LTE model in the application to the k-alpha diagnostics of hot dense matter," High Energy Density Physics 3, 57-64 (2007).

53 https://github.com/ComputationalRadiationPhysics/liblifft.

54 R. Pausch, A. Debus, R. Widera, et al., "How to test and verify radiation diagnostics simulations within particle-in-cell frameworks," Nuclear Instruments and Methods in Physics Research Section A : Accelerators, Spectrometers, Detectors and Associated Equipment 740, $250-256$ (2014).

55 M. Altarelli, "The european x-ray free-electron laser: toward an ultra-bright, high repetitionrate x-ray source,” High Power Laser Science and Engineering 3 (2015).

56 R. Mishra, P. Leblanc, Y. Sentoku, et al., "Collisional particle-in-cell modeling for energy transport accompanied by atomic processes in dense plasmas," Physics of Plasmas 20(7), 072704 (2013).

57 Steiniger, K., High Yield Optical Undulators Scalable to Optical Free-Electron Laser Operation by Traveling-Wave Thomson-Scattering. $\mathrm{PhD}$ thesis (2017). unpublished.

58 S. H. Glenzer and R. Redmer, "X-ray thomson scattering in high energy density plasmas," Rev. Mod. Phys. 81(4), 1625-1663 (2009).

59 R. Torchio, F. Occelli, O. Mathon, et al., "Probing local and electronic structure in warm dense matter: single pulse synchrotron x-ray absorption spectroscopy on shocked fe," Sci. Rep. 6, 26402 (2016).

60 J. P. Colombier, P. Combis, F. Bonneau, et al., "Hydrodynamic simulations of metal ablation by femtosecond laser irradiation," Phys. Rev. B 71, 165406 (2005). 
61 R. Ramis, J. M. ter Vehn, and J. Ramírez, "MULTI2D - a computer code for two-dimensional radiation hydrodynamics," Computer Physics Communications 180, 977-994 (2009).

62 J. J. Rehr, J. J. Kas, M. P. Prange, et al., "Ab initio theory and calculations of x-ray spectra," Comptes Rendus Physique 10, 548-559 (2009).

63 M. S. del Rio, L. Rebuffi, J. Demsar, et al., “A proposal for an open source graphical environment for simulating x-ray optics,” Proc. SPIE 9209, 9209X (2014).

64 “GNU General Public License.” http://www.gnu.org/licenses/gpl.html.

65 https://www.docker.com.

\section{List of Figures}

1 Baseline workflow in simex_platform. Green boxes represent Calculators, yellow boxes are data interfaces.

2 Schematic setup of a single particle imaging experiment

3 X-ray beam cross-section (top) on-axis power density (middle), and spectrum (bottom) at the source (left) and after propagation to focus (right).

4 X-ray laser power, average ionization, and root-mean-square displacement (rmsd) as function of time for a 9 fs FWHM x-ray pulse at $5 \mathrm{keV}$ photon energy interacting with the 2NIP protein simulated with XMDYN and XATOM.

5 Simulated diffraction intensities without (left) and with (right) radiation damage.

The sample is the 2NIP protein, photon energy $4.96 \mathrm{keV}$. The detector, an array of $80 \mathrm{x} 80$ pixels of sidelength $1.2 \mathrm{~mm}$ is placed $13 \mathrm{~cm}$ downstream from the sample. The circles mark the half-period resolution in $\AA$. 
6 "Poissonized" photon counts corresponding to the diffraction intensities in Fig. 5.

7 Schematic rendering of the proposed experiment to be simulated. Ultra-short pulsed optical laser radiation (red beam) creates a plasma layer on a metal foil's surface and accelerates electrons and ions. The XFEL beam (blue) probes the plasma perpendicular to the optical laser axis. A pixel area detector captures photons scattered in forward direction.

8 PIC simulations of the electron density (top) and forward scattering patterns (bottom) for various types of laser-plasma instabilities.

9 Block diagram for integrated simulations of $\mathrm{x}$-ray scattering from a high power laser excited plasma. 
10 SAXS signal from a laser-driven cryogenic hydrogen strand. The left image shows the electron number density distribution 134 fs after a $30 \mathrm{fs}, 105 \mathrm{TW}$ infrared driver laser pulse has hit the $5 \mu \mathrm{m}$ thick solid hydrogen strand. The laser is incident from the left and focused to a spot size of $3 \mu \mathrm{m}$ full-width at half maximum of the intensity profile. The initial hydrogen number density is thirty critical densities $n_{\mathrm{c}}=\epsilon_{0} m c^{2} k_{0}^{2} / q^{2}$ for the laser wavelength of $\lambda_{\text {Laser }}=800 \mathrm{~nm}$. During laser irradiation electrons are pushed by the laser ponderomotive force leading to hole boring and an increase in density near the laser propagation axis. The electron density distribution can be imaged by SAXS with an $\mathrm{x}$-ray pulse of femtosecond scale duration. X-ray pulse and pump laser direction of propagation are perpendicular such that the $1 \AA$ wavelength $\mathrm{x}$-ray pulse illuminates the target from the side on a $7 \times 7 \mu \mathrm{m}^{2}$ area. The right image shows a region of interest from the corresponding synthetic scattering signal on an $\mathrm{x}$-ray camera $93 \mathrm{~cm}$ behind the target. It is obtained by a Fourier transform of the two dimensional electron density data of the area illuminated by the XFEL pulse assuming that all free electrons of the $5 \mu \mathrm{m}$ thick, fully ionized target contribute to the scattering signal and that the density does not change siginificantly during XFEL pulse illumination. The incident X-ray pulse parameters match those expected for the European XFEL HED instrument ${ }^{49,55}$ which provides $10^{12}$ photons. With photon counts in the range of $10^{5}$ to $10^{6}$ per $13 \times 13 \mu \mathrm{m}^{2}$ detector pixel the scattering signal is well-measurable within a single shot which proves the feasibility to record femtosecond and nanometer scale dynamics of laser-driven plasmas at XFELs. Electron density data was obtained by a PICLS ${ }^{56}$ simulation. 
11 Schematic rendering of a laser driven shock compression experiment and $\mathrm{x}$-ray absorption spectroscopy (a). XANES signal as function of time indicating structural phase transition from bcc to hcp phase (b). 1-D Radiation-hydrodynamics simulation of the shock propagation and pressure dynamics during laser-matter interaction (c). Reproduced from Ref. ${ }^{59}$

\section{List of Tables}

1 X-ray pulse parameters for the HED instrument at the European XFEL. ${ }^{48,49}$

2 European XFEL HED PP laser parameters for optical laser-matter interaction simulations ${ }^{48,49}$

3 ESRF optical laser parameters for long pulse laser-matter simulations.

4 Principal $\mathrm{x}$-ray parameters for shock compression studies at ESRF (beamline ID24) 